\title{
Correction to: Evaluation of Predictors of Protein Relative Stability Obtained by \Solid-State Hydrogen/Deuterium Exchange Monitored by FTIR
}

\author{
Rui Fang ${ }^{1,2}$ • Wasfy Obeidat ${ }^{1,3}$ • Michael J. Pikal ' Robin H. Bogner ' \\ Published online: 17 February 2021 \\ (C) Springer Science+Business Media, LLC, part of Springer Nature 2021
}

\section{Correction to: Pharmaceutical Research volume 37,} Article number: 168 (2020)

https://doi.org/ I0.1007/s I | 095-020-02897-7

This Erratum is to add the following affiliation as a second affiliation to Dr. Wasfy Obeidat. The second affiliation is Department of Pharmaceutical Technology, Faculty of Pharmacy, Jordan University of Science and Technology, Irbid, Jordan.

The original article has been corrected.

The online version of the original article can be found at https://doi.org/l 0 . | 007/s | | 095-020-02897-7

Robin H. Bogner

Robin.bogner@uconn.edu

University of Connecticut, Storrs, CT, USA

2 Present address: Sterile and Specialty Products, Merck \& Co., Inc, Kenilworth, NJ, USA

3 Department of Pharmaceutical Technology, Faculty of Pharmacy, Jordan University of Science and Technology, Irbid, Jordan 